\title{
Charged Higgs discovery potential in the single top mode in 2HDMs
}

\author{
Renato Guedes \\ Centro de Física Teórica e Computacional, Faculdade de Ciências, Universidade de Lisboa, Av. \\ Prof. Gama Pinto 2, 1649-003 Lisboa, Portugal. \\ E-mail: renatodcii.fC.ul.pt
}

\section{Stefano Moretti}

NExT Institute and School of Physics and Astronomy, University of Southampton Highfield,

Southampton SO17 1BJ, UK.

E-mail: stetanodphys.soton.ac.uk

\section{Rui Santos*}

Instituto Superior de Engenharia de Lisboa, Rua Conselheiro Emídio Navarro 1, 1959-007

Lisboa, Portugal and

Centro de Física Teórica e Computacional, Faculdade de Ciências, Universidade de Lisboa, Av.

Prof. Gama Pinto 2, 1649-003 Lisboa, Portugal.

E-mail: rsantosdcii.fc.ul.pt

\begin{abstract}
We discuss the discovery potential of a charged Higgs boson in the single top mode. The models discussed include a CP-conserving and a CP-violating version of the softly broken $Z_{2}$ symmetric $2 \mathrm{HDM}$ potential. We conclude that the single top mode could help to constrain the $\left(m_{H^{ \pm}}, \tan \beta\right)$ plane in several versions of 2 HDMs.
\end{abstract}

Fourth International Workshop on Prospects for Charged Higgs Discovery at Colliders - CHARGED2012, October 8-11, 2012

Uppsala Sweden

${ }^{*}$ Speaker. 


\section{Introduction}

As the $8 \mathrm{TeV}$ run at the CERN's Large Hadron Collider (LHC) is reaching the end, one may ask if a light charged Higgs will survive the confrontation with experimental data in extensions of the Standard Model (SM) that allow for the existence of at least one charged Higgs boson. In models where portions of the parameter space will still survive the $8 \mathrm{TeV}$ data, is there any hope of excluding a light charged Higgs for all of the parameter space by the end of the $13-14 \mathrm{TeV}$ run? As will be clear later on, the answer to that question is no for some versions of two-Higgs doublet models (2HDMs). However, we will show that a slight improvement can nevertheless be obtained by complementing the present search, based on the $t \bar{t}$ mode, with the search in the single top mode.

\section{Two-Higgs doublet models}

CP-conserving as well as CP-violating (either explicit or spontaneous) $2 \mathrm{HDMs}$ ' potentials with a softly broken $Z_{2}$ symmetry, $\Phi_{1} \rightarrow \Phi_{1}, \Phi_{2} \rightarrow-\Phi_{2}$, can be written as

$$
\begin{aligned}
V\left(\Phi_{1}, \Phi_{2}\right) & =m_{1}^{2} \Phi_{1}^{\dagger} \Phi_{1}+m_{2}^{2} \Phi_{2}^{\dagger} \Phi_{2}+\left(m_{12}^{2} \Phi_{1}^{\dagger} \Phi_{2}+\text { h.c }\right)+\frac{1}{2} \lambda_{1}\left(\Phi_{1}^{\dagger} \Phi_{1}\right)^{2}+\frac{1}{2} \lambda_{2}\left(\Phi_{2}^{\dagger} \Phi_{2}\right)^{2} \\
& +\lambda_{3}\left(\Phi_{1}^{\dagger} \Phi_{1}\right)\left(\Phi_{2}^{\dagger} \Phi_{2}\right)+\lambda_{4}\left(\Phi_{1}^{\dagger} \Phi_{2}\right)\left(\Phi_{2}^{\dagger} \Phi_{1}\right)+\frac{1}{2} \lambda_{5}\left[\left(\Phi_{1}^{\dagger} \Phi_{2}\right)^{2}+\text { h.c. }\right]
\end{aligned}
$$

where $\Phi_{i}, i=1,2$ are complex $\mathrm{SU}(2)$ doublets.

Hermiticity of the potential forces all parameters except $m_{12}^{2}$ and $\lambda_{5}$ to be real. The choice of $m_{12}^{2}$ and $\lambda_{5}$, together with the nature of the vacuum expectation values (VEVs) will determine the CP nature of the model (see [四] for a review). This, in turn, dictates whether we end up with two CP-even Higgs states, usually denoted by $h$ and $H$, and one CP-odd state, usually denoted by $A$ (the CP-even case) or with three spinless states with undefined CP quantum number, usually denoted by $h_{1}, h_{2}$ and $h_{3}$ (the CP-violating case). However, as long as the VEVs do not break the electric charge, which was shown to be possible in any 2HDM [ฉ], there are in any case two (identical) charged Higgs boson states, one charged conjugated to the other.

In this work we will focus on two specific realisations, one CP-conserving and the other ex-

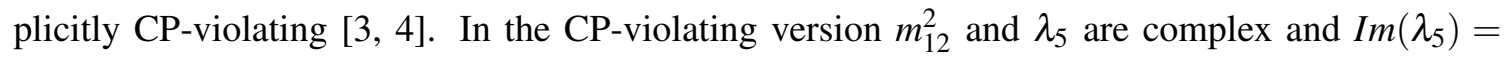
$v_{1} v_{2} \operatorname{Im}\left(m_{12}^{2}\right)$. In both models the VEVs are real. By defining $\tan \beta=v_{2} / v_{1}$, it is then possible to choose the angle $\beta$ as the rotation angle from the group eigenstates to the mass eigenstates in the charged Higgs sector. By then extending the $Z_{2}$ symmetry to the Yukawa sector we end up with four independent $2 \mathrm{HDMs}$, the well known [[6, 目] Type I (only $\phi_{2}$ couples to all fermions), Type II ( $\phi_{2}$ couples to up-type quarks and $\phi_{1}$ couples to down-type quarks and leptons), Type Y or III ( $\phi_{2}$ couples to up-type quarks and to leptons and $\phi_{1}$ couples to down-type quarks) and Type X or IV ( $\phi_{2}$ couples to all quarks and $\phi_{1}$ couples to leptons) (details and couplings can be found in [प]).

We will now very briefly discuss the main experimental and theoretical constraints affecting the 2HDM parameter space. The signal in our analysis originates from single top production with the subsequent decay $t \rightarrow b H^{ \pm} \rightarrow b \tau \nu$. Hence, only the charged Higgs Yukawa couplings are present and therefore the only parameters we need to be concerned with $\operatorname{are} \tan \beta$ and the charged Higgs mass. Values of $\tan \beta$ smaller than $O(1)$ together with a charged Higgs with a mass below $100 \mathrm{GeV}$ are disallowed both by the constraints [[]] coming from $R_{b}$, from $B_{q} \bar{B}_{q}$ mixing and from 
$B \rightarrow X_{s} \gamma$ for all models. Furthermore, data from $B \rightarrow X_{s} \gamma$ impose a lower limit of $m_{H^{ \pm}} \gtrsim 340 \mathrm{GeV}$, but only for models Type II and Type Y. The LEP experiments have set a lower limit on the mass of the charged Higgs boson of $79.3 \mathrm{GeV}$ at $95 \%$ C.L., assuming only $B R\left(H^{+} \rightarrow \tau^{+} v\right)+B R\left(H^{+} \rightarrow\right.$ $c \bar{s})=1\left[\right.$ [Q] . These bounds led us to take $m_{H^{ \pm}}>90 \mathrm{GeV}$ and $\tan \beta>1$ for type I and X. We will also present results for model type II, where the bounds on the charged Higgs mass can be evaded due to the presence of new particles as is the case of the MSSM.

\section{Results and discussion}

$p p \rightarrow t \bar{t}$ is the best process to search for a charged Higgs boson at the LHC. However, because the single top production cross section is about one third of $\sigma_{p p \rightarrow t \bar{t}}$, it certainly deserves a full investigation regarding its contribution to the production of charged Higgs bosons. The signal consists mainly of a light charged Higgs boson produced via $t$-channel graphs, $p p \rightarrow t j \rightarrow H^{+} \bar{b} j$ and $H^{+} \rightarrow \tau^{+} v$, together with $p p \rightarrow \bar{t} j \rightarrow H^{-} b j$ and $H^{-} \rightarrow \tau^{-} \bar{v}$, where $j$ represents a light-quark jet. In what follows we are considering proton-proton collisions at a center-of-mass (CM) energy of $\sqrt{s}=14 \mathrm{TeV}$ and a top-quark mass $m_{t}=173 \mathrm{GeV}$. We consider a charged Higgs boson mass interval of 90 to $160 \mathrm{GeV}$ and the analysis is performed in $10 \mathrm{GeV}$ mass steps.

Maximising the signal-to-background significance $(S / \sqrt{B})$ makes both the $s$-channel and the $t W$ single-top production modes negligible - only the $t$-channel process survives the set of cuts imposed. Signal events were generated with POWHEG [ए0]] at NLO with the CTEQ6.6M [ए]] PDFs. The top was then decayed in PYTHIA [ㅁ]. We have considered only the leptonic decays of the tau-leptons, that is, the signal final state is $p p \rightarrow l b j \not E$, where $l=e, \mu$ (electrons and muons) while $\mathbb{E}$ means missing (transverse) energy.

The irreducible background, single-top production with the subsequent decay $t \rightarrow b W^{+}$, was also generated with POWHEG. The main contributions to the reducible background are: $t \bar{t}$ production, $W^{ \pm}+$jets (including not only light quarks and gluons, but also $c$ - and $b$-quarks) and the pure QCD background ( $j j j$, where $j$ is any jet). The $t \bar{t}$ background was generated with POWHEG while $W^{ \pm}+$jets $(1,2$ and 3 jets) was generated with AlpGen [[13]]. Finally, the QCD background was generated with CalcHEP [प4]] (with CTEQ61l PDFs). The hadronisation was performed with PYTHIA 6. After hadronisation DELPHES [ㄷ], was used to simulate the detector effects. For the detector and trigger configurations, we resorted to the ATLAS default definitions.

In order to maximise $S / \sqrt{B}$ we apply the following selection cuts (see [ [] ] for details)

1. We demand one electron with $p_{T}>30 \mathrm{GeV}$ or a muon with $p_{T}>20 \mathrm{GeV}$, and $|\eta|<2.5$ for both leptons.

2. We veto events with two or more leptons with $p_{T}>10 \mathrm{GeV}$. This cut eliminates the leptonic $t \bar{t}$ background almost completely.

3. We veto events with leptons having $p_{T}$ above $55 \mathrm{GeV}$.

4. Events with missing energy below $50 \mathrm{GeV}$ are excluded. This is a cut that dramatically reduces the QCD background. 
5. We ask for one and only one $b$-tagged jet with $p_{T}<75 \mathrm{GeV}$. We assume a $b$-tagging efficiency of 0.4 (with $\mathrm{R}=0.7$ ), while the misidentification rates for the case of $c$-quark jets we take 0.1 and for lightquark/ gluon jets we adopt 0.01 .

6. We reconstruct a "top quark invariant mass" as defined in [ $[\mathbf{]}]$ and demand all events to have this invariant mass above $280 \mathrm{GeV}$.

7. We define a leptonic transverse mass [ $\left[\right.$ ],$M_{T}^{l v}$, and we have accepted events with $30 \mathrm{GeV}<$ $M_{T}^{l v}<60 \mathrm{GeV}$ for charged Higgs masses between 90 and $130 \mathrm{GeV}$ and $30 \mathrm{GeV}<M_{T}^{l v}<$ $60 \mathrm{GeV}$ or $M_{T}^{l v}>85 \mathrm{GeV}$ for higher values of the charged Higgs mass.

8. We have chosen events with one and one jet (non- $b$ ) only with $p_{T}>30 \mathrm{GeV}$ and $|\eta| \leq 4$.9.

9. We veto all events with a jet multiplicity equal to two or above for jets with $p_{T}>15 \mathrm{GeV}$ and $|\eta| \leq 4.9$.

10. We only accept events where jets have a pseudorapidity $|\eta| \geq 2.5$.

Putting all the numbers together we can find $S / B$ and $S / \sqrt{B}$ as a function of the charged Higgs mass as presented in table $\mathbb{T}$.

\begin{tabular}{ccccc}
\hline$m_{H}^{ \pm}(\mathrm{GeV})$ & Signal $(S)$ & Background $(B)$ & $S / B(\%)$ & $S / \sqrt{B}$ \\
\hline 90 & 38.6 & 29.5 & 130.92 & 7.11 \\
100 & 40.5 & 29.5 & 137.19 & 7.45 \\
110 & 45.6 & 29.8 & 153.00 & 8.35 \\
120 & 47.7 & 30.1 & 158.26 & 8.69 \\
130 & 42.3 & 32.68 & 129.53 & 7.41 \\
140 & 117.1 & 77.9 & 150.25 & 13.26 \\
150 & 120.0 & 86.6 & 138.64 & 12.90 \\
160 & 109.7 & 100.8 & 108.81 & 10.92 \\
\hline
\end{tabular}

Table 1: Signal-to-Background ratio $(S / B)$ and significance $(S / \sqrt{B})$ as a function of the charged Higgs mass for $\sqrt{s}=14 \mathrm{TeV}$ and a luminosity of $1 \mathrm{fb}^{-1}$. The numbers presented for the signal we take $\mathrm{BR}\left(t \rightarrow b H^{ \pm}\right)=$ $100 \%$ and $\mathrm{BR}\left(H^{-} \rightarrow \tau^{-} v\right)=100 \%$ and all other BRs have the usual SM values.

The results can be presented in a model independent manner $[\mathbb{}[]$ and exclusion plots can be derived for the different 2HDMs. In figure $\square$ we present the exclusion plots for the MSSM (left) and Type $\mathrm{X}$ (right) in the $\left(\tan \beta, m_{H^{ \pm}}\right)$plane at the $95 \% \mathrm{CL}$ assuming the $\mathrm{LHC}$ at $14 \mathrm{TeV}$ and for several luminosity sets. The results show similar trends to the ones obtained for $t \bar{t}$ production. We started with a cross section that is about three times smaller than the $t \bar{t}$ one and ended up with a result that is 2 to 3 times worse than the prediction presented by ATLAS [ए6]. It is expectable that both ATLAS and CMS will improve the results on the single top mode presented here, improving the constraints on the $\left(m_{H^{ \pm}}, \tan \beta\right)$ plane. One may now ask what are the chances to probe the entire $\left(m_{H^{ \pm}}, \tan \beta\right)$ plane by the end of the $14 \mathrm{TeV}$ run. In view of the results for $7 \mathrm{TeV}$ [ए], [8]], one expects a type II charged Higgs to be excluded by then. However, there are models where the Yukawa couplings always decrease with $\tan \beta$ as is the case of models I and X. For those models, 

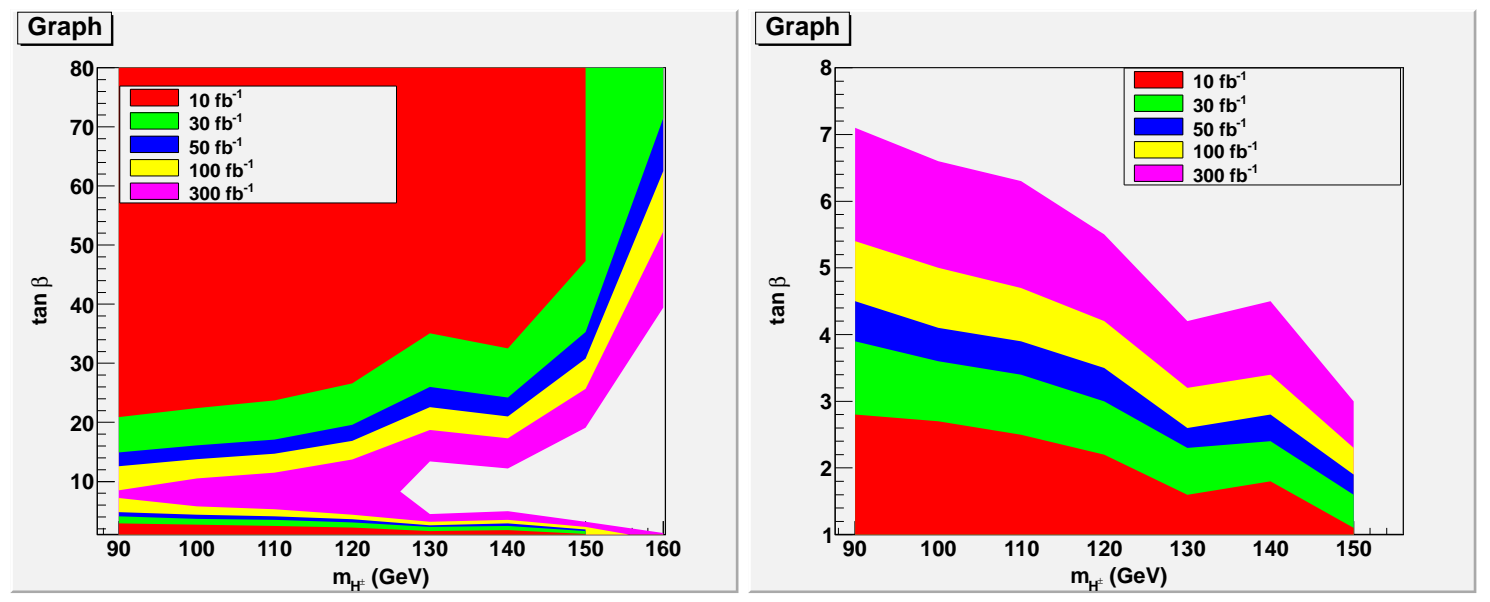

Figure 1: The MSSM (left) and Type X (right) exclusion limits over the $\left(\tan \beta, m_{H^{ \pm}}\right)$plane at the $95 \% \mathrm{CL}$ assuming the $\mathrm{LHC}$ at $14 \mathrm{TeV}$ and for several luminosity sets.

we know that $p p \rightarrow t \bar{t}$ will provide the strongest constraint on the $\left(m_{H^{ \pm}}, \tan \beta\right)$ plane, and that the single top mode is bound to contribute even if only with a slight improvement. Are there any other processes that could help to probe the large $\tan \beta$ region?

There is another Yukawa process, $c s \rightarrow H^{ \pm}(+j e t)$ [प्व, [20], that could in principle help to probe the above mentioned region. It was however shown to be negligible for large $\tan \beta$. The remaining possibility [20] is to look for processes that either do not depend on $\tan \beta$, or even better, that grow with $\tan \beta$. There are terms both in $g g \rightarrow H^{+} W^{-}$and in Vector Boson Fusion $\left(p p \rightarrow j j H^{+} H^{-}\right.$where $j$ is a light quark jet) that are independent of $\tan \beta$. Furthermore, for the CP-conserving potential, there is a term in $g g \rightarrow H^{+} H^{-}$that has the form

$$
\sigma_{p p \rightarrow H^{+} H^{-}} \propto \sin (2 \alpha) \tan \beta\left(m_{H}^{2}-M^{2}\right)
$$

where $\alpha$ is the rotation angle in the CP-even sector, $m_{H}$ is the heavier CP-even scalar mass and $M^{2}=m_{12}^{2} /(\sin \beta \cos \beta)$. Hence, there are regions of the $2 \mathrm{HDM}$ parameter space that can be probed for larger values of $\tan \beta$. However, the bounds will no longer be for a two parameter space but instead for a multi-dimension space with all $2 \mathrm{HDM}$ parameters playing a role. Further, values of the cross section that could lead to meaningful significances, are only obtained for resonant production. Therefore, only a small portion of the multidimensional space can be probed for large $\tan \beta$ (see [20] for details).

A final comment about theoretical bounds. Assuming that the Higgs boson was discovered with a mass of $125 \mathrm{GeV}$, it was recently shown in [R]] that for the particular case of an exact CPconserving $Z_{2}$ symmetric model $\tan \beta<6$. Therefore, that particular model will probably see a light charged Higgs ruled out when all the $8 \mathrm{TeV}$ data is analysed.

Acknowledgments SM is financed in part through the NExT Institute. The work of RG and RS is supported in part by the Portuguese Fundação para a Ciência e a Tecnologia (FCT) under contracts PTDC/FIS/117951/2010 and PEst-OE/FIS/UI0618/2011. RG is also supported by a FCT Grant SFRH/BPD/47348/2008. RS is also partially supported by an FP7 Reintegration Grant, number PERG08-GA-2010-277025. 


\section{References}

[1] G. C. Branco, P. M. Ferreira, L. Lavoura, M. N. Rebelo, M. Sher and J. P. Silva, Phys. Rept. 516 (2012) 1.

[2] P. M. Ferreira, R. Santos and A. Barroso, Phys. Lett. B 603 (2004) 219 [Erratum-ibid. B 629 (2005) 114].

[3] I. F. Ginzburg, M. Krawczyk and P. Osland, hep-ph/0211371.

[4] A. W. El Kaffas, W. Khater, O. M. Ogreid and P. Osland, Nucl. Phys. B 775 (2007) 45.

[5] V. D. Barger, J. L. Hewett and R. J. N. Phillips, Phys. Rev. D 41 (1990) 3421.

[6] M. Aoki, S. Kanemura, K. Tsumura and K. Yagyu, Phys. Rev. D 80 (2009) 015017.

[7] R. Guedes, S. Moretti and R. Santos, JHEP 1210 (2012) 119.

[8] F. Mahmoudi and O. Stal, Phys. Rev. D 81, 035016 (2010); F. Mahmoudi, talk given at Prospects For Charged Higgs Discovery At Colliders (CHARGED 2012), 8-11 October, Uppsala, Sweden; M. Misiak, H. M. Asatrian, K. Bieri, M. Czakon, A. Czarnecki, T. Ewerth, A. Ferroglia and P. Gambino et al., Phys. Rev. Lett. 98, 022002 (2007); D. Asner et al. [Heavy Flavor Averaging Group Collaboration], arXiv:1010.1589 [hep-ex]; A. Wahab El Kaffas, P. Osland and O. Magne Ogreid, Phys. Rev. D 76 (2007) 095001; L. Basso, A. Lipniacka, F. Mahmoudi, S. Moretti, P. Osland, G. M. Pruna and M. Purmohammadi, arXiv:1205.6569 [hep-ph].

[9] LEP Higgs Working Group for Higgs boson searches, ALEPH, DELPHI,L3 and OPAL Collaborations, arXiv: hep-ex/0107031.

[10] S. Alioli, P. Nason, C. Oleari and E. Re, JHEP 1006 (2010) 043; S. Alioli, P. Nason, C. Oleari and E. Re, JHEP 0909 (2009) 111 [Erratum-ibid. 1002 (2010) 011]; E. Re, Eur. Phys. J. C 71 (2011) 1547; S. Frixione, P. Nason and G. Ridolfi, JHEP 0709 (2007) 126; P. Nason, JHEP 0411 (2004) 040; S. Frixione, P. Nason and C. Oleari, JHEP 0711 (2007) 070.

[11] P. M. Nadolsky et al., Phys. Rev. D 78 (2008) 013004.

[12] T. Sjostrand, S. Mrenna and P. Z. Skands, JHEP 0605 (2006) 026.

[13] M. L. Mangano, M. Moretti, F. Piccinini, R. Pittau and A. D. Polosa, JHEP 0307 (2003) 001.

[14] A. Pukhov, arXiv:hep-ph/0412191.

[15] S. Ovyn, X. Rouby and V. Lemaitre, arXiv:0903.2225 [hep-ph].

[16] G. Aad et al. [ATLAS Collaboration], arXiv:0901.0512 [hep-ex].

[17] G. Aad et al. [ATLAS Collaboration], JHEP 1206 (2012) 039.

[18] S. Chatrchyan et al. [CMS Collaboration], JHEP 1207 (2012) 143.

[19] H. J. He and C. P. Yuan, Phys. Rev. Lett. 83 (1999) 28; S. R. Slabospitsky, arXiv:hep-ph/0203094.

[20] M. Aoki, R. Guedes, S. Kanemura, S. Moretti, R. Santos and K. Yagyu, Phys. Rev. D 84 (2011) 055028; R. Guedes, S. Kanemura, S. Moretti, R. Santos and K. Yagyu, PoS CHARGED 2010 (2010) 037.

[21] B. Gorczyca, M. Krawczyk, arXiv:1112.5086 [hep-ph]. 